\title{
Analysis of Flow Dynamics in the High-Flux Gas-Solid Riser Using Trajectory Distances across Attractors Reconstructed from Solid Concentration Signals
}

\author{
Justin M. Jeremiah1', Samwel V. Manyele1, Abraham K. Temu'1, Jesse-X. Zhu² \\ ${ }^{1}$ Department of Chemical and Mining Engineering, University of Dar es Salaam, Dar es Salaam, Tanzania \\ ${ }^{2}$ Department of Chemical and Biochemical Engineering, University of Western Ontario, London, ON, Canada \\ Email: jeremiahjmj@udsm.ac.tz,smanyele@udsm.ac.tz, atemu@udsm.ac.tz,jzhu@uwo.ca
}

How to cite this paper: Jeremiah, J.M., Manyele, S.V., Temu, A.K. and Zhu, J.-X. (2018) Analysis of Flow Dynamics in the High-Flux Gas-Solid Riser Using Trajectory Distances across Attractors Reconstructed from Solid Concentration Signals. Engineering, 10, 688-703.

https://doi.org/10.4236/eng.2018.1010050

Received: May 30, 2018

Accepted: October 20, 2018

Published: October 23, 2018

Copyright $\odot 2018$ by authors and Scientific Research Publishing Inc. This work is licensed under the Creative Commons Attribution-NonCommercial International License (CC BY-NC 4.0). http://creativecommons.org/licenses/by-nc/4.0/ cc) (i) (8) Open Access

\begin{abstract}
The study of the entrance and wall dynamics of a high-flux gas-solid riser was conducted using trajectory distances of the reconstructed attractors from solid concentration signals collected from a $76 \mathrm{~mm}$ internal diameters and 10 $\mathrm{m}$ high riser of a circulating fluidized bed (CFB) system. The riser was operated at 4.0 to $10.0 \mathrm{~m} / \mathrm{s}$ gas velocity and 50 to $550 \mathrm{~kg} / \mathrm{m}^{2} \mathrm{~s}$ solids flux. Spent fluid catalytic cracking (FCC) catalyst particles with $67 \mu \mathrm{m}$ mean diameter and density of $1500 \mathrm{~kg} / \mathrm{m}^{3}$ together with $70 \%$ to $80 \%$ humid air was used. Solid concentration data were analyzed using codes prepared in FORTRAN 2008 to get trajectories of the reconstructed attractors and their distances apart. Trajectory distances were found to increase from the centre towards the wall indicating the expansion of the attractor. The probability density function (PDF) of the trajectory distances changes from single peak at the centre to multiple peaked profiles in the wall region. Multiple peaked profiles indicate multifractal flow behaviours. Cumulative distribution functions (CDF) of the trajectory distances changes from single $S$-shaped at the centre to multiple $S$-shaped profiles in some locations of the wall region indicating multifractal flow behaviours. The PDF distribution of these distances at the entrance section and in the wall region forms different types of statistical distributions showing differences in gas-solid flow structures in various spatial locations of the wall region and the entrance sections. Most of the distributions at the centre fall under the Gumbel max distribution for all flow development sections of the riser, especially at air velocities of $5.5 \mathrm{~m} / \mathrm{s}$ and $8 \mathrm{~m} / \mathrm{s}$ showing uniform flow structures. Further, it was found that increase of the number of the phase space reconstruction embedding dimension increases the trajectory distances between the state vectors leading to the expansion of
\end{abstract}


the attractor.

\section{Keywords}

Trajectory Distance, Probability Density Function, Cumulative Distribution Function, Euclidian Distance, High-Flux Riser

\section{Introduction}

Fluidization technology is extensively used in various processes in which large contacts between the solid and fluid phases during reaction is required [1] [2]. Fluidized bed reactors such as the circulating fluidized bed (CFB) risers are used in the chemical industries, fossil fuels industries such as petroleum and coal, metallurgical industries, food and pharmaceutical industries, etc. [3] [4] [5]. Fluidized bed reactors are highly preferred in these processes as they provide high contacting efficiency and good control of the feed materials. However, despite of the tremendous advantages over many other reactors, their successful operations require a better understanding of their dynamics [3] [4].

There various methods which are used to investigate the gas-solid flow dynamics in circulating fluidized bed risers such as statistical, frequency and chaos analysis methods [3]. However, chaos analysis is significantly preferred in studying the gas-solid flow dynamics in risers due to its ability to account its non-linear and complex behaviours. The method involves collection of a single variable time series data and embeds it to determine state vectors which are used to reconstruct attractors in an embedded phase space. Then invariant parameters such as correlation dimensions, Lyapunov exponents and kolmogorov entropy are determined and used to study the complexity of the reconstructed attractor [3].

Chaos analysis begins with reconstruction of attractors which is achieved by embedding the measured single variable time series using time delay and sufficient minimum embedding dimension. This step is very importance due to the fact that most of chaotic parameters used in characterizing the dynamical system depends on the correctness of the reconstruction process and the accuracy of the reconstructed attractors [6]. Once the attractor is established, its invariant parameters which describe its characteristics are computed and used to describe the dynamic behaviours of the system from which the time series signal is measured.

The use of statistical analysis of the vector pair distances referred to as trajectory distances has been used in describing the dynamics of high-flux gas-solid riser using pressure fluctuation signals [7]. However, the use of this technique for solid concentration data obtained from high-flux gas-solid riser is not reported. This study extend this technique by using the probability density and cumulative distribution functions of the trajectory distances of the pair vectors on attractors reconstructed from solid concentration signals measured from 
high-flux gas-solid circulating fluidized bed (CFB) riser.

\section{Literature Review}

\subsection{Phase Space Analysis}

The phase space or state space is a space spanned by dependent variables of a given dynamical system in which all possible states of a system are represented, with each possible state of the system corresponding to one unique point in the phase space [8] [9] [10]. A phase space represents a dynamical system where each point on that space represents a particular state of the system at a particular time. Thus, analysis of such system requires reconstruction of an attractor in a phase space from which its dynamics can be studied through non-linear time series methods using a measured signal of one variable [3] [11].

The analysis of chaotic dynamic system makes use of the property that it must be sensitive to initial conditions [8]. The sensitivity to initial conditions is explained in a way each point in a chaotic system is arbitrarily closely approximated by other points with significantly different future paths, or trajectories. This property is utilized in the embedding theory to mimic the original phase space. Thus a very small change to the current trajectory may lead to significant different future behaviour which is explained as a high rate of information loss with time [8]. The change in trajectories paths can be utilized to study the change in dynamics. The property of topological mixing or transitivity explains how the system evolves over time so that any given region or open set of its phase space eventually overlap with any other given region. Also the property of having dense periodic orbits points to the phenomena that every point in the space is approached arbitrarily closely by periodic orbits [8].

Due to the fact that the phase space determine all states of a dynamical system, analysis of that system can be achieved in both identifying the system and predicting the future states via phase space representation [9]. Specifying a point in the phase space specifies the state of the system. Thus, by studying the dynamics of trajectory points on the phase space like the change in their distances enables to quantify the dynamics of the system [9] [10].

\subsection{Phase Space Representation}

The most challenging point in chaos analysis is the failure to understand the original phase space of the dynamical system. Thus the analysis achieved by using embedded or reconstructed phase space. The embedded space enables to draw out a multidimensional description of state space dynamics from time series data of a single dynamical variable, and generalizes the quantitative measures of a chaotic behaviour [9] [10].

According to Takens' embedding theorem it is possible to reconstruct phase space from a single time series so as to characterize a non-linear dynamical system through time delayed variables [12]. The embedding theorems developed by Takens and then Sauer et al. states that "if the sequence $\left(x_{N}\right)$ does indeed consist 
of scalar measurements of the state of a dynamical system, then under certain genericity assumptions, the time delay embedding provides a one-to-one image of the original set $(X)$, provided the embedding dimension $(m)$ is large enough" [13]. A time-delayed variable constitute an adequate embedding, provided the measured variable is smooth and couples to all other variables [10].

Further, it is possible to reconstruct the phase space attractor while preserving the topological characteristics from measurements of a single dynamical variable. This renders experimental systems, like CFB reactors with attractors of moderately low dimension, acceptable for analysis by chaos analysis tools. This idea was introduced by Packard et al. in 1980 [14] and the mathematical proof of the validity of this idea is due to Takens [12].

The reconstructed attractor consists of orbits of points corresponding to different state vectors, $X(i)$, which represents one point on the orbit of the attractor and is reconstructed from the data points of the time series. Reconstruction of the state vectors is achieved by the method of delays where a time delay, $\tau$, of the time steps between the elements of the state vector and the number of elements, $m$, of the state vector equals to the embedding dimensions are chosen and transposed to create a multidimensional state vector [10] [13] [15]. Suppose the measured time series data of single variable $x(1), x(2), \ldots, x(N)$, is embedded using the time delay, $\tau$, and the embedding dimension, $m$, then the resulting state vectors of the attractor is given by Equation (1)

$$
X(i)=[x(i), x(i+\tau) x(i+2 \tau), \cdots, x(i+(m-1))]^{\mathrm{T}}
$$

where the count, $i=1,2,3, \ldots, N$.

\section{Methodology}

\subsection{Data Collection}

Data were collected from a CFB system shown in Figure 1 with a twin-riser having 76 and $203 \mathrm{~mm}$ internal diameters and $10 \mathrm{~m}$ high operated at 50 to 550 $\mathrm{kg} / \mathrm{m}^{2} \mathrm{~s}$ solids flux and 4.0 to $10.0 \mathrm{~m} / \mathrm{s}$ gas velocity. Fluid catalytic cracking catalyst particles with $67 \mu \mathrm{m}$ mean diameter and density of $1500 \mathrm{~kg} / \mathrm{m}^{3}$ were used. A $70 \%$ to $80 \%$ humid air was used for transporting the solid particles. Signals were sampled from eight (8) axial levels (i.e., $Z=0.98,1.52,2.73,3.96,5.13,6.34,8.74$, and $9.42 \mathrm{~m}$ ) and 11 radial points (i.e., $r / R=0.00,0.16,0.38,0.50,0.59,0.67,0.74$, $0.81,0.87,0.92$, and 0.98 ) at each level where $r / R$ is the normalized radial distances from the center to the wall of the riser. To each point, 29,100 data points of solid concentration were sampled in 30 seconds using optical fiber probe at $970 \mathrm{~Hz}$.

\subsection{Determination of Trajectories Distances (Euclidean Distance)}

The trajectory distances of the state vectors from the reconstructed attractor with time delay, $\tau=6$ and embedding dimension, $m=15$ were determined using a multi-dimensional Euclidian distances formula, used in chaos analysis. The 


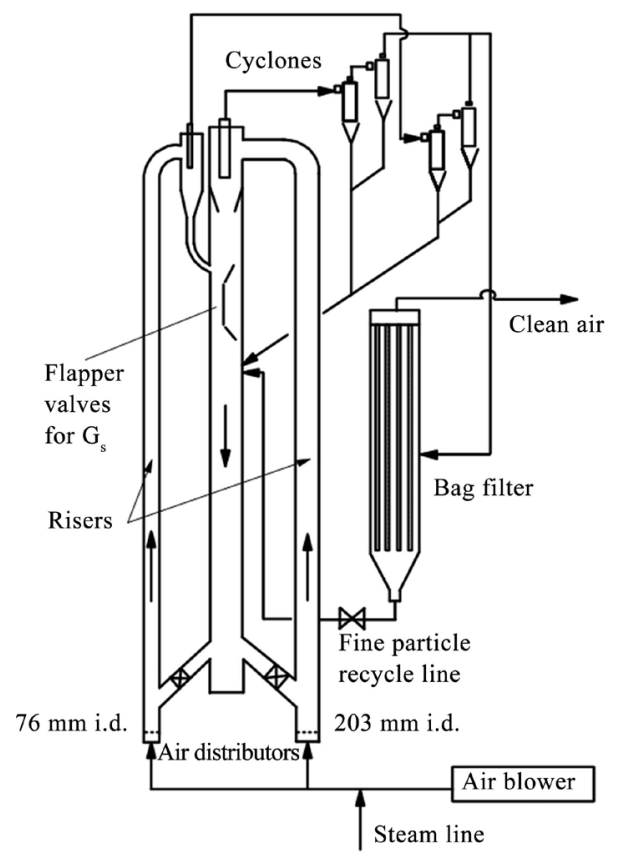

Figure 1. The circulating fluidized bed with two riser system.

trajectory distances of the state vectors were calculated as per Equation (2) [11] [16] [17]:

$$
\delta_{i j}=\left\|X_{i}-X_{j}\right\|
$$

where $\left\|X_{i}-X_{j}\right\|$ is the vector distance (Euclidean distance) between two reconstructed vectors, $X_{\mathrm{i}}$ and $X_{\mathrm{j}}$. The Euclidian distance, $\delta$, is the vector distance between vector pairs $\left(X_{\mathrm{i}}, X_{\mathrm{j}}\right)$ of the trajectories. The vector distances (Euclidean distance), $\delta$, was calculated using Equation (3) as follows [16] [18]:

$$
\delta_{i j}=\sum_{i=1}^{N} \sum_{j=i+1}^{N} \sqrt{\left(X_{i}-X_{j}\right)^{2}}
$$

This quantity was determined on the reconstructed attractors using signals from different radial locations near the wall along the riser to study the chaotic behaviour of the high-flux riser. These distances from each signal were calculated and saved in the file. In this work the number of solid concentrations data points, $N=2000$. The distances between the pairs $\left(X_{\mathrm{i}}, X_{\mathrm{j}}\right)$ were computed by first keeping $i$ constant at $i=1$ and then navigating all points, $j=i+1$ to $N$, and then $i$ was changed from $i=1$ to $N$ [18]. This lead to the size of time series data used to determine trajectory distances equals to $N t s=N^{2}=4 \times 10^{6}$, the computation which were achieved using prepared code in FORTARN 2008.

\subsection{Establishment of PDF for Trajectory Distances}

To establish the PDF of the trajectory distances, the data sets were first split into bins of equal intervals. This was achieved by subtracting the minimum value from the maximum value and dividing the obtained range by the set number of steps. The obtained interval was used to establish various bins starting with the 
one that included the minimum data value and get subsequent bins until the maximum data value was included.

Suppose $\delta$ is the distance separating any two points or trajectories on the attractor and $N$ is the number of points counted on the attractor along the trajectories, then the PDF was established by setting equal intervals of $\delta, \Delta \delta$, between $\delta_{\min } \leq \delta \leq \delta_{\max }$ by choosing 50 steps to make 50 bins and counting number of points within the distance $\delta_{m i n} \leq \delta \leq \delta_{p}$ that is, $N_{i}$.

The frequency for each interval, $\Delta \delta$, was determined as per Equation (4)

$$
f_{i}=\frac{N_{i}}{N t s} \times 100 \%
$$

For count, $i=1,2,3, \ldots$

By changing to next interval $\delta_{i} \leq \delta \leq(i+1) \Delta \delta$ until the last bin the frequency table was created and the frequency curve plotted which gives the PDF of the values of $\delta$ across the attractor. The cumulative distribution function was established where the cumulative frequency was determined by adding each frequency to the sum of its preceding frequency as per Equation (5):

$$
c f_{i}=f_{i-1}+f_{i}
$$

For count, $i=1,2,3, \ldots$

The shapes of PDF, CDF and their location along $\ln (\delta)$-axis were used to study the changes in the flow dynamics of the gas-solid riser.

\section{Results and Discussion}

\subsection{Sample Probability Density and Cumulative Distribution Functions}

The probability density functions (PDF) describe the relative likelihood for the random variable to take on a given value and the cumulative distribution function $(\mathrm{CDF})$ is a function whose value is the probability that a variable takes a value less than or equal to the argument of the function. In this work the trajectory distances of the state vectors of the reconstructed attractors were determined and the PDF and the CDF of these distances were established and their profiles plotted.

Figure 2 shows the plots of the Probability Density Function (PDF) profiles in the first raw and the Cumulative Distribution Functions (CDF) profiles in the second raw from the fully developed flow section (Axial elevation, $Z=9.42 \mathrm{~m}$ ) of the riser in the wall region at different radial position for gas velocity, $U_{\mathrm{g}}=5.5$ $\mathrm{m} / \mathrm{s}$ and solid flux, $G_{\mathrm{s}}=300 \mathrm{~kg} / \mathrm{m}^{2} \mathrm{~s}$.

When different PDF from different signals are examined critically, their shapes like tails, height and number of peaks can be used get information on the behaviours of the gas-solid flow dynamics, spatial locations or operating conditions of the gas velocity and solid flux. A uniform gas-solid suspension flow gives a trajectory distances close to the mean distance and leads to the formation of single, narrow and tall PDF. Example of this is found in PDF profiles at $r / R=$ 


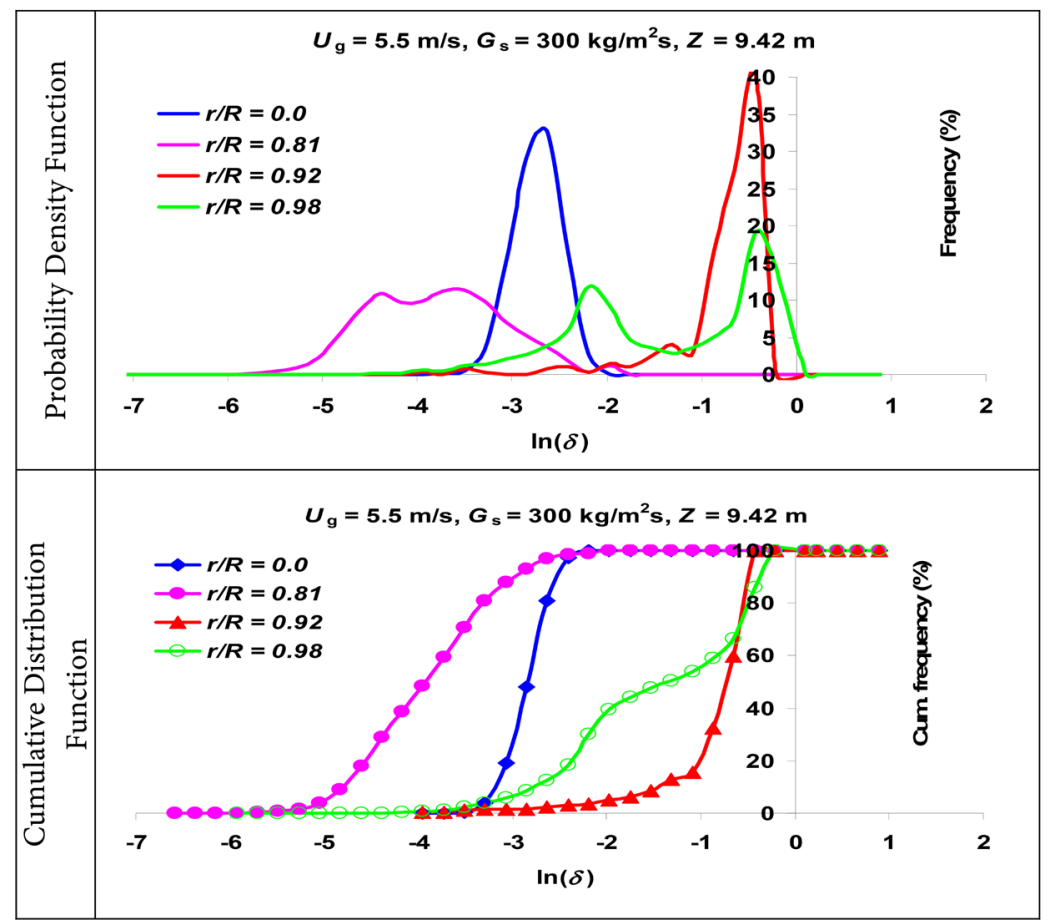

Figure 2. Sample probability and cumulative distributions functions.

0.0 as shown in Figure 2. Towards the wall as shown for $r / R=0.81,0.92$ and 0.98 profiles has two or more peaks indicating presence of bifractal or multifractal flow behaviours. A more segregated suspension gives a wider and flatter PDF which are cases found in the wall region.

Also different CDF generated from different signals display differences in shapes and number of $S$-shape in the CDF profiles. These features were used in this study to differentiate the behaviours of the gas-solid flow dynamics, spatial locations and or the operating conditions of the gas velocity. In Figure 2 the CDF at the centre $r / R=0.0$ have single $S$-shaped profile. But, for $r / R=0.98$ the CDF has double $S$-shape indicating presence of bifractal flow behaviour at that particular location. However, some of the features may not be seen using the CDF or PDF only especially for microstructure flow behaviour. For instance the PDF at $r / R=0.81$ reveal double peaks which indicate bifractal behaviour. This behaviour is not revealed using the CDF from the same location where it show single $S$-shaped profile indicating single flow behaviour.

This study introduces another way of examining the gas-solid flow behaviours in the circulating fluidized bed riser using PDF and CDF of the trajectory distances from the reconstructed attractors. Further this work introduces new planes, that is, the frequency-ln $(\delta)$ plane and the cumulative frequency-ln $(\delta)$ plane.

\subsection{Probability Density Functions of the Trajectory Distances across Reconstructed Attractor in the Wall Region}

The PDF of the trajectory distances of the reconstructed attractor reveals the 
scattering tendency of these distances in the phase space. For the trajectory distances data to be compared with the correlation integral data or curves, it was suggested to use same scale, that is $\ln (\delta)$, which can be mapped on the $\ln (r)$, and hence locate the distances on such axis, originally developed by Grassberger and Procaccia [19]. Thus, the trajectory distances on the reconstructed attractor were determined using a multi-dimensional Euclidian distances formula. The distances were logged into a file, followed by statistical analysis. Figure 3 shows probability density function (PDF) of natural logarithms of the trajectory distances at 5.5, 8.0 and $10 \mathrm{~m} / \mathrm{s}$ when $G_{\mathrm{s}}=300 \mathrm{~kg} / \mathrm{m}^{2} \mathrm{~s}$ for selected riser heights, $z=$ $1.52,3.96$ and $9.42 \mathrm{~m}$, corresponding to riser entrance, developing flow and fully developed flow sections.

Results shows that for $U_{g}=5.5 \mathrm{~m} / \mathrm{s}$ the trajectory distances or the pair spacing between trajectories vector increases from the centre towards the wall especially in the developing section $(Z=3.96 \mathrm{~m})$. Multiple peaks of the profiles for instances at $Z=9.42 \mathrm{~m}$ and $r / R=0.98$ indicates the presence of multifractal flow structures.

When the gas velocity, $U_{g}=8.0 \mathrm{~m} / \mathrm{s}$, Figure 3 shows that the trajectory distances between trajectory vectors increase from the centre of the riser towards the wall. This is well depicted in the entrance region $(Z=1.52 \mathrm{~m})$, flow developing section $(Z=3.96 \mathrm{~m})$ and in the fully developed section $(Z=9.42 \mathrm{~m})$ especially

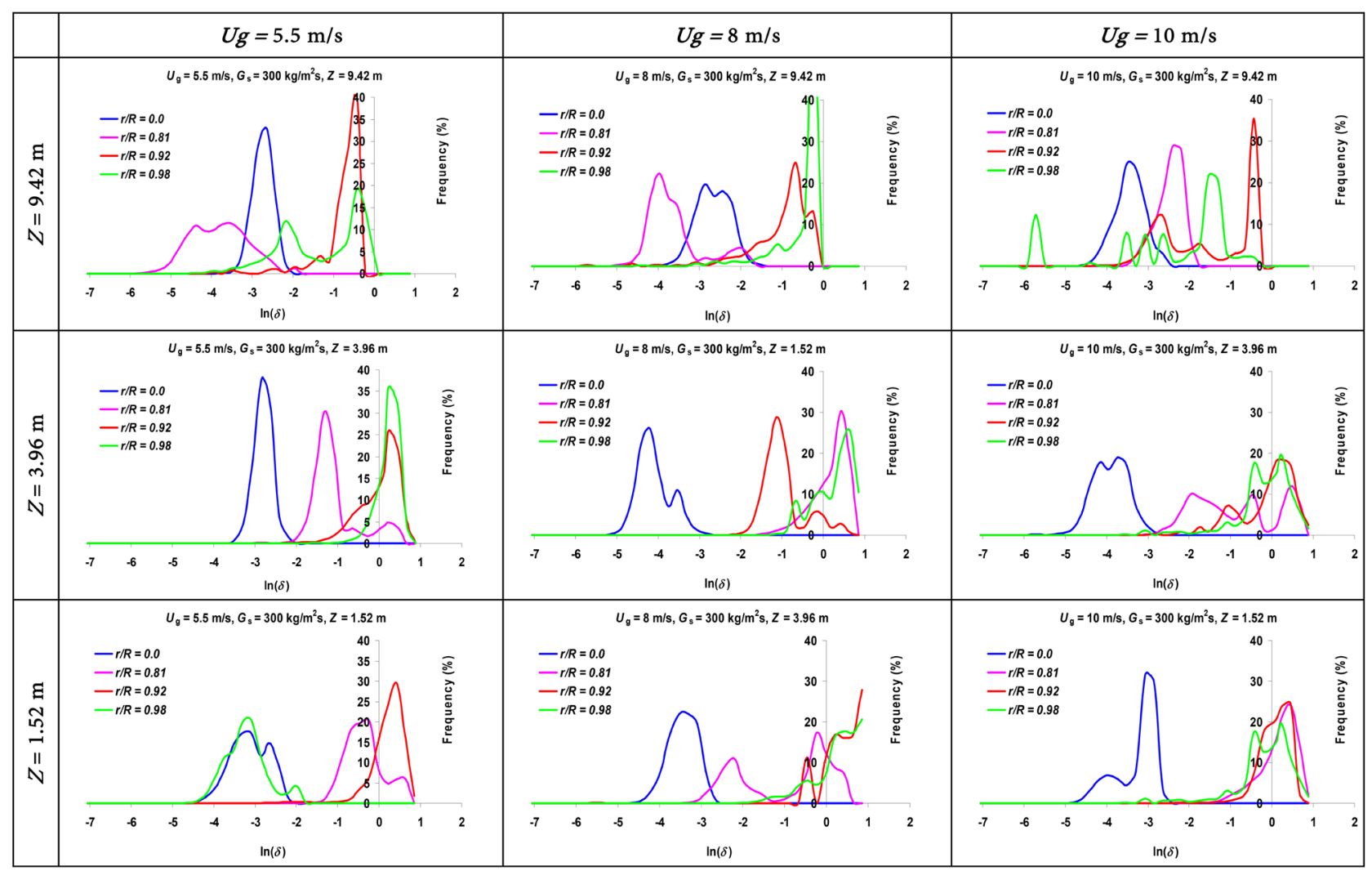

Figure 3. Probability density functions of natural logarithms of the trajectory distances at $5.5,8.0$ and $10 \mathrm{~m} / \mathrm{s}$ when $G_{\mathrm{s}}=300$ $\mathrm{kg} / \mathrm{m}^{2}$ s for $z=1.52,3.96$ and $9.42 \mathrm{~m}$, corresponding to the riser entrance, developing flow and fully developed flow sections respectively. 
in the developing section $(Z=3.96 \mathrm{~m})$. Presence of more than one peak in some profiles for instances profiles at $Z=9.42 \mathrm{~m}$, at $Z=3.96$ and $r / R=0.81$ indicates a multifractal flow structures, reported also in [3].

When the gas velocity, $U_{g}=10 \mathrm{~m} / \mathrm{s}$, Figure 3 results show a clear increase of the trajectory distances between vectors from the centre of the riser towards the wall. This is well depicted in all sections of the riser. In all sections close to the wall, at $r / R=0.98$, the profiles shows multiple peaks. This indicates a distinct multifractal flow structures. However, there is also tendency to form double peaked PDF at the center, i.e., $r / R=0.0$, in the entrance section $(Z=1.52 \mathrm{~m})$ for all velocities, i.e., $U_{\mathrm{g}}=5.5,8$ and $10 \mathrm{~m} / \mathrm{s}$. This tendency is more clear at low velocity, $U_{\mathrm{g}}=5.5 \mathrm{~m} / \mathrm{s}$. At this velocity the center profiles $(r / R=0.0)$ in the developing flow and fully developed flow sections has single peaked PDF while at $U_{\mathrm{g}}$ $=8 \mathrm{~m} / \mathrm{s}$ the PDF in the fully developed section $(Z=9.42 \mathrm{~m})$ the profile is double peaked. At higher velocity, $U_{\mathrm{g}}=10 \mathrm{~m} / \mathrm{s}$, the PDF profiles close to the wall, i.e., at $r / R=0.92$ and 0.98 have higher tendency to form multiple peaks compared to the profiles at $U_{\mathrm{g}}=8$ and $5.5 \mathrm{~m} / \mathrm{s}$. As the axial elevation, $Z$, increase from the entrance section to the top section, the PDF profiles at $r / R=0.81$ shifts towards the negative direction for all velocities, i.e., $U_{\mathrm{g}}=5.5,8$ and $10 \mathrm{~m} / \mathrm{s}$. Further, it can be seen that the center profiles $(r / R=0.0)$ are located towards lower values of $\ln (\delta)$ in the negative direction while profiles closer to the wall (i.e., $r / R=0.92$ and $0.98)$ are located towards higher values of $\ln (\delta)$ in the positive direction around the centre of the Frequency $(\%)-\ln (\delta)$ plane.

The dynamic study of the riser using PDF of the trajectory distances is reported in literature [15]. However, this study used pressure fluctuation signal which accounted for the change in dynamics along the axial elevations of the riser. Using pressure fluctuation signals could not be used to show the dynamic variations along the radial direction. This study has used solid concentration signals from different locations along the radial and axial directions through which variations in radial and axial dynamics have been analysed. The shape of the PDF in 15] did not show the presence of the multiple peaks which has been shown in this study to account for multifractal flow structures.

\subsection{Types of Probability Density Functions Profiles and Their Parameters from the Trajectory Distances}

The probability density functions of the trajectory distances were further analysed to determine their distribution type for further characterization of gas-solid flow behaviours. The best fitting distribution were achieved using Easy Fit 5.3 Professional software. The software gives three tests of the goodness of fit, i.e., the Kolmogorov Smirnov, Anderson Darling and the Chi-square. The Kolmogorov Smirnov was chosen in this study where the statistics and the ranking for each of the fitted distribution are given. The first rank were taken as the best fit for the distribution and hence parameters were recorded as shown in Table 1 where the type of distribution and parameters of the trajectory distances in the entrance section $(Z=1.52 \mathrm{~m})$, developing section $(Z=3.96 \mathrm{~m})$ and the devel- 
oped section $(Z=9.42 \mathrm{~m})$ for $U_{\mathrm{g}}$, $=5.5,8$ and $10 \mathrm{~m} / \mathrm{s}$.

From Table 1 it can be observed that all trajectory distances at the center fall under Gumbel Max distribution except in the entrance and developing sections when $U_{\mathrm{g}}=10 \mathrm{~m} / \mathrm{s}$ where the uniform distribution emerges. This indicates that the flow behaviours at the center have almost similar behaviour. In the wall region the trajectory distances gives different PDF distributions including the normal, beta, hypersecant and wake by distributions. The difference in distribution type indicates presence of different gas-solid flow behaviours in the wall region.

\subsection{Cumulative Distribution Function Profiles of the Trajectory Distances}

The cumulative distribution function (CDF) shows the probability that the variable takes a value less than or equal to the argument of the function. In this case, $\mathrm{CDF}$ shows the probability that the $\ln (\delta)$ or $\delta$ takes a value less than (ogive) or equal to the frequency (\%) indicated. Figure 4 shows the cumulative distribution

Table 1. Probability density function distribution of the trajectory distances in the entrance, developing and developed sections for $U_{\mathrm{g}}=5.5,8$ and $10 \mathrm{~m} / \mathrm{s}$.

\begin{tabular}{|c|c|c|c|c|c|c|c|c|c|}
\hline \multirow{2}{*}{$Z(\mathrm{~m})$} & \multirow{2}{*}{$\begin{array}{l}U_{\mathrm{g}} \\
(\mathrm{m} / \mathrm{s})\end{array}$} & \multicolumn{2}{|l|}{$r / R=0.0$} & \multicolumn{2}{|l|}{$r / R=0.81$} & \multicolumn{2}{|l|}{$r / R=0.92$} & \multicolumn{2}{|l|}{$r / R=0.98$} \\
\hline & & Distribution & Parameters & Distribution & Parameters & Distribution & Parameters & Distribution & Parameters \\
\hline \multirow{6}{*}{9.42} & \multirow{2}{*}{5.5} & \multirow[t]{2}{*}{ Gumbel Max } & $\sigma=5.2693$ & \multirow[t]{2}{*}{ Gumbel Max } & $\sigma=2.8732$ & \multirow{2}{*}{ Normal } & $\sigma=7.1255$ & \multirow{2}{*}{ Normal } & $\sigma=4.0424$ \\
\hline & & & $\mu=-1.0423$ & & $\mu=0.34164$ & & $\mu=2.0$ & & $\mu=2.0$ \\
\hline & \multirow[b]{2}{*}{8} & \multirow[b]{2}{*}{ Gumbel Max } & $\sigma=4.0105$ & \multirow[b]{2}{*}{ Normal } & $\sigma=4.8721$ & \multirow[b]{2}{*}{ Power Function } & $\alpha=0.07565$ & \multirow{2}{*}{ Logistic } & $\sigma=4.9405$ \\
\hline & & & $\mu=-0.31331$ & & $\mu=2.0024$ & & $\begin{array}{l}a=7.1852 \mathrm{E}-15 \\
b=43.293\end{array}$ & & $\mu=2.001$ \\
\hline & \multirow{2}{*}{10} & \multirow{2}{*}{ Gumbel Max } & $\sigma=4.3473$ & \multirow{2}{*}{ Gumbel Max } & $\sigma=4.8334$ & \multirow{2}{*}{ Hypersecant } & $\sigma=5.5092$ & \multirow{2}{*}{ Normal } & $\sigma=4.723$ \\
\hline & & & $\mu=-0.51067$ & & $\mu=-0.79036$ & & $\mu=2.0005$ & & $\mu=2.0013$ \\
\hline \multirow{12}{*}{3.96} & \multirow{4}{*}{5.5} & \multirow{4}{*}{ Gumbel Max } & & \multirow{4}{*}{ Normal } & & \multirow{4}{*}{ Wakeby } & $\alpha=\beta=0$ & \multirow{4}{*}{ Wakeby } & $\alpha=\beta=0$ \\
\hline & & & $\sigma=3.7447$ & & $\sigma=5.7161$ & & $\gamma=0.45539$ & & $\gamma=0.22683$ \\
\hline & & & $\mu=-0.16135$ & & $\mu=2.0016$ & & $\delta=0.79149$ & & $\delta=0.89205$ \\
\hline & & & & & & & $\zeta=-0.18323$ & & $\zeta=-0.1013$ \\
\hline & \multirow{4}{*}{8} & \multirow{4}{*}{ Gumbel Max } & & & $\alpha=\beta=0$ & & & & $\alpha=\beta=0$ \\
\hline & & & $\sigma=4.381$ & Wakeby & $\gamma=0.96022$ & Gumbel Max & $\sigma=4.5165$ & Wakeby & $\gamma=0.54882$ \\
\hline & & & $\mu=-0.53154$ & wakeoy & $\delta=0.58642$ & Gumbel Max & $\mu=-0.60692$ & Wakeby & $\delta=0.75212$ \\
\hline & & & & & $\zeta=-0.32036$ & & & & $\zeta=-0.21321$ \\
\hline & & & & & & & $\alpha_{1}=0.02333$ & & $\alpha_{1}=0.05168$ \\
\hline & & & $a=-6.7745$ & & $a=-3.9335$ & & $\alpha_{2}=0.44276$ & & $\alpha_{2}=0.12406$ \\
\hline & 10 & Unitorm & $b=10.776$ & Unitorm & $b=7.935$ & Kumaraswamy & $a=-3.9090 \mathrm{E}-15$ & Beta & $a=3.7075 \mathrm{E}-15$ \\
\hline & & & & & & & $b=29.725$ & & $b=19.7$ \\
\hline & & Gumbel Max & $\sigma=3.7447$ & & & & $K=0.84788$ & & $a=-6.3847$ \\
\hline & 5.5 & 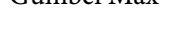 & $\mu=-0.16135$ & Gumbel Max & $\mu=-0.26051$ & Gen. Logistic & $\sigma=0.31815$ & Uniform & $b=10.389$ \\
\hline & & & & & & & $\mu=0.20292$ & & \\
\hline & 8 & Gumbel Max & $\sigma=3.7447$ & Uniform & $a=7.9596$ & Normal & $\sigma=5.6584$ & Gumbel Max & $\sigma=4.1329$ \\
\hline 1.52 & $\mathrm{o}$ & & $\mu=-0.16135$ & Sintoniti & $b=11.96$ & IVoninal & $\mu=1.9998$ & Guntuel tiad & $\mu=-0.38528$ \\
\hline & & & & & & & $\alpha=\beta=0$ & & $\alpha_{1}=0.05168$ \\
\hline & 10 & Uniform & $a=-8.7682$ & Uniform & $a=-7.2731$ & Wakeby & $\gamma=0.36001$ & Beta & $\alpha_{2}=0.12406$ \\
\hline & 10 & Umorm & $b=12.769$ & Uminorm & $b=11.275$ & wakeoy & $\delta=0.83294$ & Deta & $a=3.7075 \mathrm{E}-15$ \\
\hline & & & & & & & $\zeta=-0.15385$ & & $B=19.7$ \\
\hline
\end{tabular}




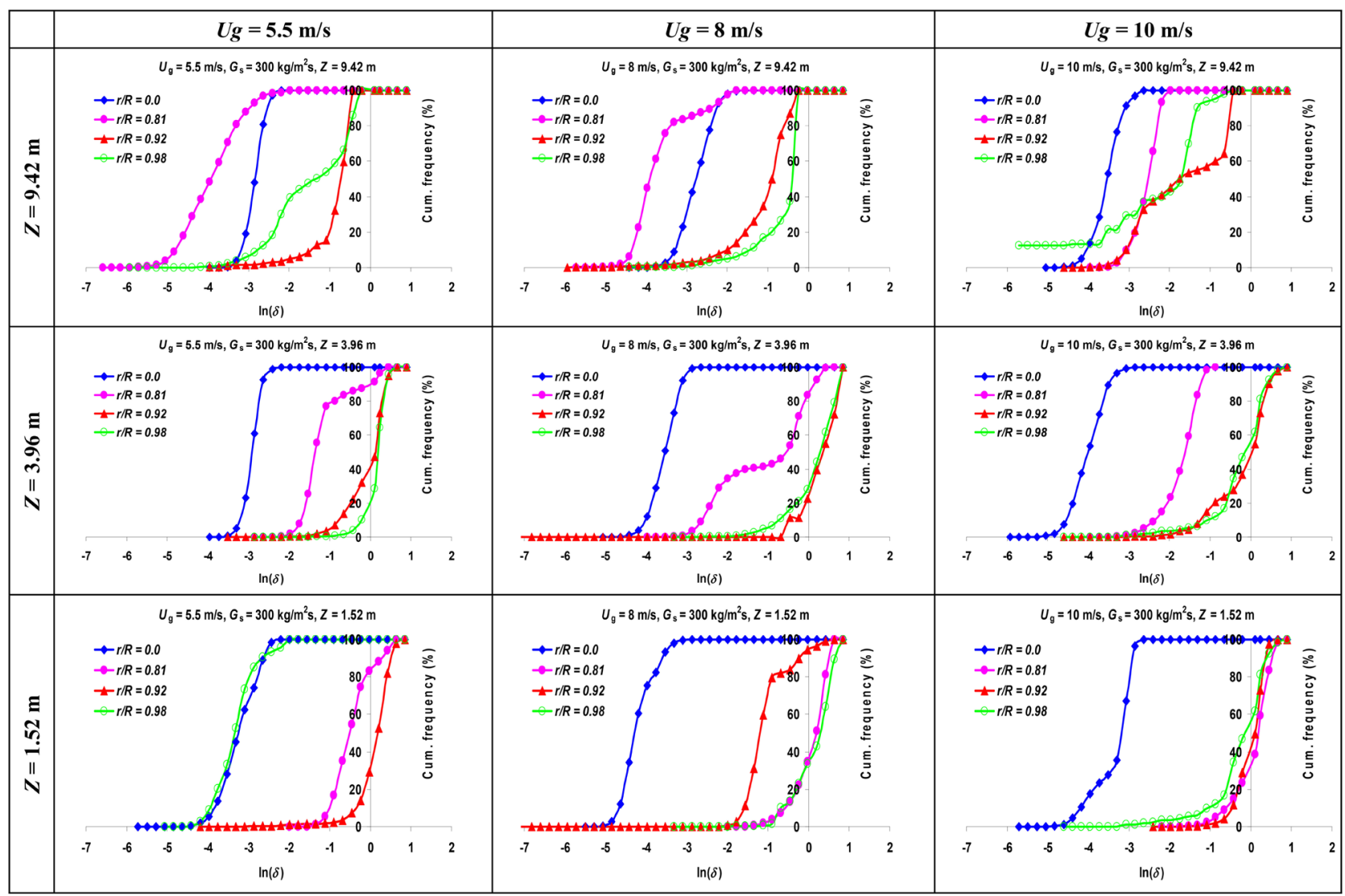

Figure 4. Cumulative distribution curves for $\ln (\delta)$ for solid concentration signals from selected radial positions in the wall region and along different axial elevations along the riser at $U_{\mathrm{g}}=5.5,8.0$ and $10 \mathrm{~m} / \mathrm{s}$ for $G_{\mathrm{s}}=300 \mathrm{~kg} / \mathrm{m}^{2}$ s.

function curves for $\ln (\delta)$ for distances between points in the reconstructed attractor using solid concentration signals from selected radial positions for gas velocity, $U_{g}=5.5,8.0$ and $10 \mathrm{~m} / \mathrm{s}$ when solid flux, $G_{\mathrm{s}}=300 \mathrm{~kg} / \mathrm{m}^{2}$ s for selected riser heights, $Z=1.52,3.96$ and $9.42 \mathrm{~m}$, corresponding to riser entrance, developing flow and fully developed flow sections.

When $U_{g}=5.5 \mathrm{~m} / \mathrm{s}$ most of the profiles are single $S$ shaped with few exception at $\mathrm{Z}=9.42 \mathrm{~m}$ and $r / R=0.98$ where a double $S$ shaped CDF was observed as shown in Figure 4.

For $U_{\mathrm{g}}=8 \mathrm{~m} / \mathrm{s}$ and $G_{\mathrm{s}}=300 \mathrm{~kg} / \mathrm{m}^{2} \mathrm{~s}$ most of the profiles are single $S$ shaped with few exceptions with double $S$ shaped like the profile at $Z=3.96 \mathrm{~m}$ and $r / R=$ 0.81 also at $Z=9.42$ and $r / R=0.81$. Furthermore, Figure 4 shows that when $U_{\mathrm{g}}$ $=10 \mathrm{~m} / \mathrm{s}$ and $G_{\mathrm{s}}=300 \mathrm{~kg} / \mathrm{m}^{2} \mathrm{~s}$ also most of the profiles are single $S$ shaped with few exceptions with double $S$ shaped like the profile at $Z=9.42$ and $r / R=0.81$. Presence of the multiple S-shaped profiles indicates a distinct multifractal flow structure or mixed flow structure.

The CDF profiles shows features which are found for all velocities in various sections of the riser. For instance, the double $S$-shaped CDF is clearly observed at $r / R=0.98$ in the developed section $(Z=9.42 \mathrm{~m})$ when $U_{\mathrm{g}}=5.5 \mathrm{~m} / \mathrm{s}$ and $G_{\mathrm{s}}=$ $300 \mathrm{~kg} / \mathrm{m}^{2} \mathrm{~s}$. This kind of profiles is also found when $U_{\mathrm{g}}=8 \mathrm{~m} / \mathrm{s}$ and $G_{\mathrm{s}}=300$ 
$\mathrm{kg} / \mathrm{m}^{2} \mathrm{~s}$ for instance in the developed section (at $r / R=0.81, Z=9.42 \mathrm{~m}$ ), developing section (at $r / R=0.81, Z=3.96 \mathrm{~m}$ ) and in the entrance section at $r / R=$ 0.92 and $Z=1.52 \mathrm{~m}$. Also for $U_{\mathrm{g}}=10 \mathrm{~m} / \mathrm{s}$ and $G_{\mathrm{s}}=300 \mathrm{~kg} / \mathrm{m}^{2}$ s double $S$-shaped profile is also found in the developed section $(Z=9.42 \mathrm{~m})$ at $r / R=0.92$, in the developing section $(Z=3.96)$ at $r / R=0.92$ and in the entrance section $(Z=1.52$ $\mathrm{m})$ at $r / R=0.98$. However, at this high velocity close to the wall in the developed section $(Z=9.42)$ the profile at $r / R=0.98$ is multiple $S$-shaped.

Results show the location of some of the profiles to shift along the $\ln (\delta)$ axis particularly in the profiles in wall region. The tendency to shift the location along the $\ln (\delta)$ axis is seen for instance when $U_{\mathrm{g}}=5.5 \mathrm{~m} / \mathrm{s}, \mathrm{CDF}$ at $r / R=0.81$ shifts towards the negative direction i.e., towards lower values of $\ln (\delta)$ from the entrance section towards the top sections of the riser. However, close to the wall at $r / R=0.98$ the profile in almost similar location with the center profile $(r / R=$ $0.0)$ while in the developing section $(Z=3.96 \mathrm{~m})$ it shifts towards the positive direction, i.e., towards higher values of $\ln (\delta)$. In the developed flow section it shifts back towards the negative direction at lower values of $\ln (\delta)$. Also this behaviour is observed when $U_{\mathrm{g}}=8$ and $10 \mathrm{~m} / \mathrm{s}$, where the profiles at $r / R=0.81$ shifts towards the negative direction from the entrance section towards the developed section.

Further observations show the span or width of a dense attractor in the sphere space for some of the radial positions to change. This is revealed through the change in the span of the rising part of the CDF. For instance, when the $U_{\mathrm{g}}=5.5$ $\mathrm{m} / \mathrm{s}$, the span of the profiles at $r / R=0.98$ in the developed section $(Z=9.42 \mathrm{~m})$ is higher compared to the span of the CDF in the same radial position in the entrance $(Z=1.52)$ and developing sections $(Z=3.96)$. This behaviour is also observed when $U_{\mathrm{g}}=10 \mathrm{~m} / \mathrm{s}$ for the profiles in the wall region at $r / R=0.92$ and 0.98 .

Results in Figure 4, shows notable differences as a result of variations in operating velocities. First, when $U_{\mathrm{g}}=10 \mathrm{~m} / \mathrm{s}$ the wall region profiles $(r / R=0.81$, 0.92 and 0.98$)$ in the entrance section $(Z=1.52 \mathrm{~m})$ are close together with nearly similar shape. This however is not seen in the same section of the riser for lower velocities. However, when $U_{\mathrm{g}}=8 \mathrm{~m} / \mathrm{s}$ the only close profiles with nearly similar shape are at $r / R=0.92$ and 0.98 . When $U_{\mathrm{g}}=5.5 \mathrm{~m} / \mathrm{s}$ the wall region profiles are apart with varying shapes. Secondly, in the entrance section $(Z=1.52 \mathrm{~m})$ the center CDF profile $(r / R=0.0)$ has an $S$-shape with two linear part having different slopes. This behaviour is only observed for higher velocity, $U_{\mathrm{g}}=10 \mathrm{~m} / \mathrm{s}$. Thirdly, with exception of the CDF profiles at $r / R=0.81$ for $U_{\mathrm{g}}=5.5$ and $8 \mathrm{~m} / \mathrm{s}$ the wall region CDF profiles $(r / R=0.81,0.92$ and 0.98$)$ for all operating velocities are located to the higher values of $\ln (\delta)$ relatively to the center profile $(r / R=$ $0.0)$ which are located to the lower values of $\ln (\delta)$ along the $\ln (\delta)$ axis. Further, at higher velocities, i.e., $U_{\mathrm{g}}=10 \mathrm{~m} / \mathrm{s}$, the formation of multiple $S$-shaped profile is seen close to the wall $(r / R=0.98)$ in the developed section $(Z=9.42 \mathrm{~m})$. Multiple $S$-shaped CDF profiles are not seen for lower velocities.

Again use of the CDF of the trajectory distances is reported in literature which 
used pressure fluctuation signal that could not account for the dynamic variations along the radial direction [15]. But also the shape of the CDF in [15] could not show the single and multiple $S$-shaped structure which has been shown in this study to account for multifractal flow structures. The extension of this techniques to the solid concentration signals from different locations along the radial and axial directions of the high-flux gas-solid riser has lead the analysis of dynamic variations in all direction of the riser possible.

\subsection{Effect of Phase Space Reconstruction Dimension on the Trajectory Distances}

The phase space dimension is the smallest dimension of the space in which a phase portrait is reconstructed where the trajectory does not cross itself. The number of elements of the state vector, which equals the number of coordinates in state space, is called embedding dimensions [6] [14]. The higher enough embedding dimension gives a sufficient number of dimensions required for the attractor to expand and unfolds itself thereby giving all necessary information of the system [6] [14]. Low embedding dimension leads to contraction of the reconstructed attractor thereby obscuring some of the important information of the system. The effect of embedding dimensions on the trajectory distances was investigated for $m=1,5,10,15$ and 20. Figure 5 and Figure 6 show the probability density function of the trajectory distances at different phase space dimension at the centre $(r / R=0.92)$ and the wall region $(r / R=0.92)$.

In Figure 5 the PDF in the centre is mostly characterized by single peak despite of increasing embedding dimension especially from $m \geq 10$. The differences occur in the width of the PDF indicating the expansion of the attractor as the embedding dimension increases. Close to the wall PDF shows common behaviours for $m \geq 5$ though the width increases also indicating expansion of the attractor. This indicates that all flow behaviours manifested by the gas-solid suspensions are revealed from $m \geq 5$. Multiple peaks indicate multifractal flow behaviours which are shown for all embedding dimensions, $m \geq 5$.

From Figure 5 and Figure 6 it is observed that as the number of phase space dimension increases the PDF profiles of the trajectory distances becomes wider with the widest profiles at $m=20$. This indicates that the trajectories becomes scattered as the number of dimensions increases which indicates that the attractor expands in size. As the attractor expands it unfolds itself giving out hidden information about the flow dynamics. This shows the important of finding the sufficient minimum number of embedding for which all information about the system is unveiled leading to capturing all necessary characteristics of the flow dynamics of the CFB riser. Further at the wall $(r / R=0.92)$ the PDF shows multiple peaks which indicates high variation of the distances of the trajectories as compared to the centre which shows multifractal behaviours.

\section{Conclusion}

The entrance and wall dynamics of a high-flux gas-solid riser were studied using 
probability density functions and cumulative distributions functions of trajectory distances of the state vectors from the reconstructed attractors using solid concentration signals. This work has established the role of PDF and CDF in studying the gas-solid flow behaviour. The gas-solid flow behaviours at the wall region is well described and differentiated using PDF and CDF of the trajectory distances from the reconstructed attractor. The trajectory distances increase from the centre towards the wall indicating expansion of the attractor. The PDF of trajectory distances forms double and multiple peaks in wall region to signify

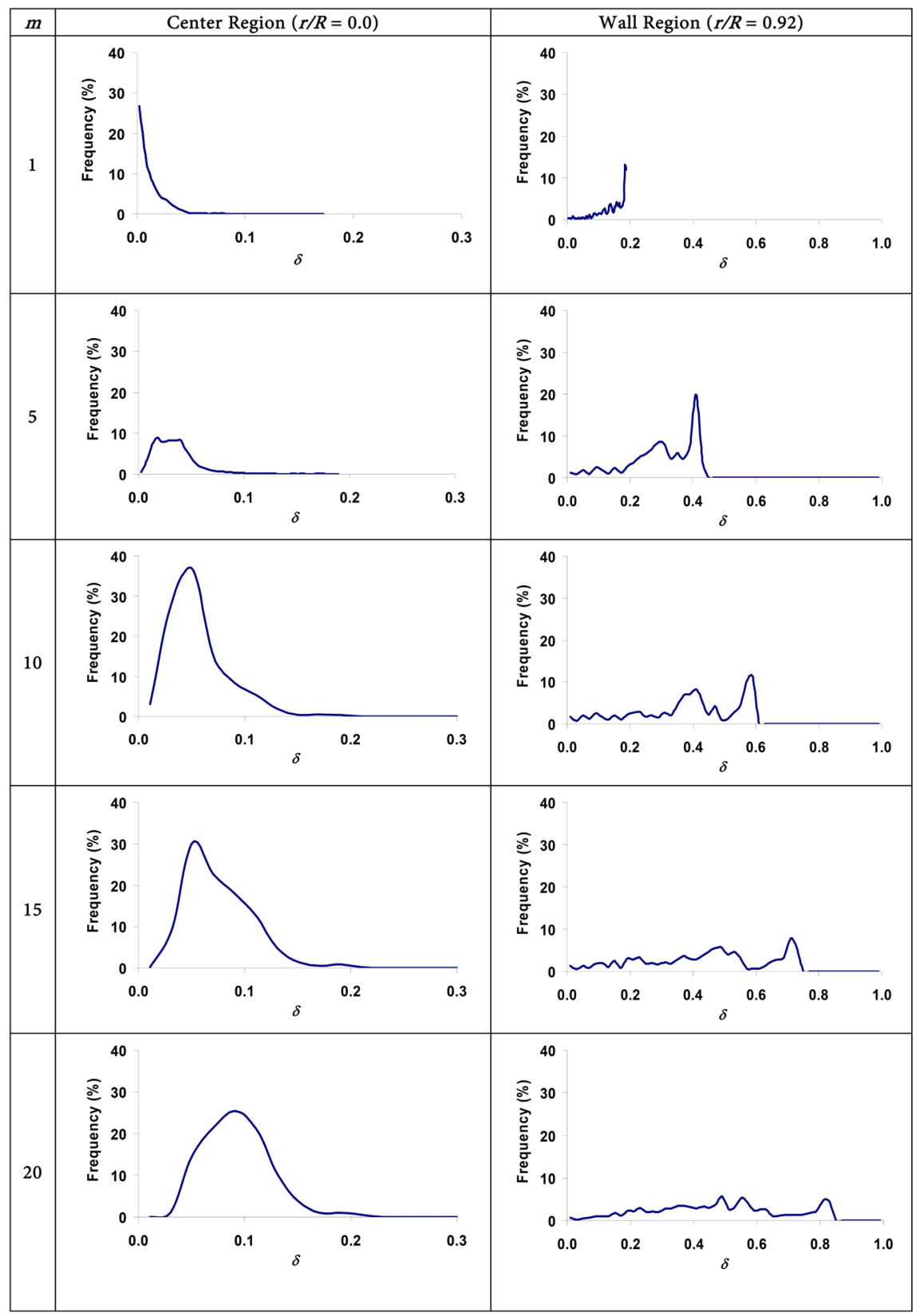

Figure 5. Comparison of the probability density function curves of the trajectory distances at different phase space dimension for $U_{\mathrm{g}}=8 \mathrm{~m} / \mathrm{s}$ and $G_{\mathrm{s}}=300 \mathrm{~kg} / \mathrm{m}^{2} \mathrm{~s}$ at $r / R=0.0$ and 0.92 for $Z=9.42 \mathrm{~m}$. 


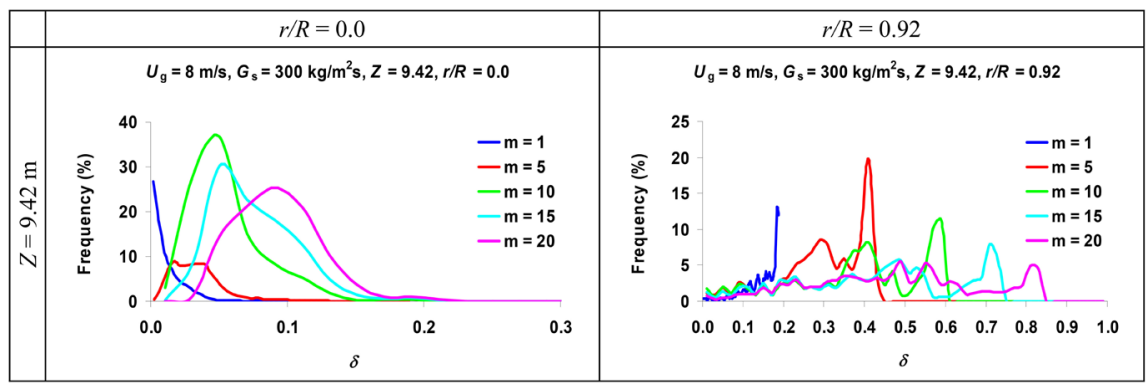

Figure 6. Comparison of the probability density function curves of the trajectory distances at different phase space dimension for $U_{\mathrm{g}}=8 \mathrm{~m} / \mathrm{s}$ and $G_{\mathrm{s}}=300 \mathrm{~kg} / \mathrm{m}^{2} \mathrm{~s}$ at $r / R=0.0$ and 0.92 for $Z=9.42 \mathrm{~m}$.

presence of bifractal and multifractal behaviours. These behaviours are presented by double and multiple $S$-shaped CDF profiles. The PDF distribution of these distances at the entrance section and in the wall region forms various types of statistical distribution showing differences in gas-solid flow structures in various spatial locations of the wall region and the entrance sections. At the centre most of the distributions fall under the Gumbel max distribution for all flow development sections of the riser, especially at lower velocities of $5.5 \mathrm{~m} / \mathrm{s}$ and 8 $\mathrm{m} / \mathrm{s}$ velocities showing uniform flow structures. In the wall region the PDF distributions shows different types in all flow development sections of the riser showing multifractal flow structures. Further, the increase in number of the phase space reconstruction dimension increases the trajectory distances between state vectors leading to the expansion of the attractor.

\section{Conflicts of Interest}

The authors declare no conflicts of interest regarding the publication of this paper.

\section{References}

[1] Ahuja, P., Agrawal, H., Sethi, A.K. and Raj, U. (2005) Chaotic Analysis of Pressure Fluctuations in a Gas-Solid Fluidized Bed. Indian Journal of Chemical Technology, 12, 212-219.

[2] Reguly Jr., H., Zinani, F., Indrusiak, M.L.S. and da Fonseca, C.E. (2015) Spectral Analysis of Pressure Fluctuations in Fluidized Beds under Different Regimes-A Numerical Study. IV Journeys in Multiphase Flows (JEM 2015), Campinas, SP, Brazil, 23-27 March 2015, 1-11.

[3] Manyele, S.V., Zhu, J.-X., Khayat, R.E. and Pärssinen, J.H. (2006) Analysis of the Chaotic Dynamics of a High-Flux CFB Riser Using Solids Concentration Measurements. China Particuology, 4, 136-146. https://doi.org/10.1016/S1672-2515(07)60253-X

[4] de Castilho, G.J. and Cremasco, M.A. (2012) Comparison of Downer and Riser Flows in a Circulating Bed by Means of Optical Fiber Probe Signals Measurement. Procedia Engineering, 42, 295-302. https://doi.org/10.1016/j.proeng.2012.07.420

[5] Cocco, R., Karri, S.B.R. and Knowlton, T. (2014) Introduction to Fluidisation Technology. American Institute of Chemical Engineers, New York. 
[6] Nichols, J.M. and Nichols, J.D. (2001) Attractor Reconstruction for Non-Linear Systems: A Methodological Note. Mathematical Biosciences, 171, 21-32. https://doi.org/10.1016/S0025-5564(01)00053-0

[7] Manyele, S.V., Khayat, R.E. and Zhu, J.-X. (2002) Investigation of the Dynamics of a High-Flux CFB Riser Using Chaos Analysis of Pressure. Chemical Engineering Technology, 25, 801-810. https://doi.org/10.1002/1521-4125(20020806)25:8<801::AID-CEAT801>3.0.CO;2-F

[8] Singh, P.P. and Handa, H. (2012) Various Synchronization Schemes for Chaotic Dynamical Systems (A Classical Survey). International Journal of Scientific Engineering and Technology, 3, 29-33.

[9] Kantz, H. and Shreiber, T. (2004) Nonlinear Time Series Analysis. 2nd Edition, Cambridge University Press, Cambridge, UK.

[10] Sevil, H.E. (2006) On the Predictability of Time Series by Metric Entropy. MSc Thesis, Izmir Institute of Technology, Izmir.

[11] Manyele, S.V., Zhu J.-X. and Zhang, H. (2003) Analysis of the Microscopic Flow Structure of A CFB Downer Reactor Using Solids Concentration Signals. International Journal of Chemical Reactor Engineering, 1, 1-17. https://doi.org/10.2202/1542-6580.1108

[12] Takens, F. (1981) Detecting Strange Attractors in Turbulence. In: Rand, D. and Young, L.S., Eds., Dynamical Systems and Turbulence, Warwick 1980. Lecture Notes in Mathematics, Vol. 898, Springer, Berlin, Heidelberg. https://doi.org/10.1007/BFb0091924

[13] Haro, A., Limaico, C. and Llosas, Y. (2015) Characterization of the Atmospheric Dynamics in Riobamba City Using the Chaos Theory. Atmospheric and Climate Sciences, 5, 441-449. https://doi.org/10.4236/acs.2015.54036

[14] Packard, N., Crutchfield, J., Farmer, D. and Shaw, R. (1980) Geometry from a Time Series. Physical Review Letters, 45, 712. https://doi.org/10.1103/PhysRevLett.45.712

[15] Johnsson, J., Zijerveld, R.C., Schouten, J.C., van den Bleek, C.M. and Leckner, B. (2000) Characterization of Fluidization Regimes by Time-Series Analysis of Pressure Fluctuations. International Journal of Multiphase Flow, 26, 663-715. https://doi.org/10.1016/S0301-9322(99)00028-2

[16] Zeng, X. (1992) Chaos Theory and Its Application in the Atmosphere. Atmospheric Science Paper No. 504.

[17] Strozzi, F., Tenrreiro, E.G., Noè, C., Rossi, T., Serati, M. and Comenges, J.Z. (2007) Application of Non-Linear Time Series Analysis Techniques to the Nordic Spot Electricity Market Data. Serie Tecnologia, 11, 1-51.

[18] Shang, P., Li, X. and Kamae, S. (2005) Chaotic Analysis of Traffic Time Series. Chaos, Solitons and Fractals, 25, 121-128. https://doi.org/10.1016/j.chaos.2004.09.104

[19] Grassberger, P. and Procaccia, I. (1983) Measurement of Strangeness of the Strange Attractors. Physica, 9D, 189-208. 